\title{
Three-dimensional DC resistivity method for real- time monitoring the lagging water inrush from floor
}

\author{
Zhixin Liu, Ran Lu \\ School of Resource and Geoscience \\ CUMT \\ Xuzhou, China \\ liuzhx@cumt.edu.cn
}

\begin{abstract}
The lag of water inrush from goaf floor is a great threat to the safety in production, which often leads to a serious property loss. A three-dimensional DC resistivity method,based on the mechanism and the inducing factors of water inrush,is proposed to monitor the possible water inrush accident in this paper. The theoretical objective function that meets the requirement of the three-dimensional DC electric field in the mine geological environment is deduced from the basic theory of stable electricity flow field. And the distribution characteristics, as well as the the changing rules of the abnormal potential caused by different spatial positions geological anomalies are studied through the approach of numerical simulation so as to provide a theoretical basis for a real-time dynamic monitoring of water inrush from the goaf floor.
\end{abstract}

Keywords—-three dimensional DC resistivity method; dynamic monitoring; lagging water-inrush;

\section{INTRODUCTION}

Because of the characteristics of lag, the water inrush from goaf floor is hidden and sudden, which is great harm to the safety of mine production. On the one hand, the lag is caused by the destruction of the original stress balance, and the development of the cracks in the floor strata. Which lead to impermeable layer breaking in a fragile surface, so that the water in the aquifer can be poured into the mined out area under hydraulic pressure. On the other hand, the small concealed structure is affected by the mining effect, and the formation of the water conducting channel is formed, so the water flowing along this water conducting channel into the goaf[1-2]. At present, the commonly used methods of mine geophysical has achieved good application effect in finding out the hidden water structure or local water area in the working face, roof and floor, and the both sides in mining influence range. Among all kinds of exploration methods, the DC resistivity method has been playing an important role for its specific advantages. With the development of full space electric field theory under the influence of roadway and forward and inverse algorithm, DC method is widely used in the prediction of floor water inrush[3-5]. However, most of these are static detection methods after the formation of the working surface, At the same time, some of the small structures and local fracture can not be effectively identified for the interpretation accuracy, During the mining process, it can be further developed into the water conducting channel affected by mining action, which can lead to the lagging water inrush from the goaf floor. To solve this problem, we hereby put forward a real-time dynamic monitoring of water inrush in working face method, the three-dimensional DC electrical method with a fixed source, provide a reference basis for safe mining.

\section{THEORY}

\section{A. Monitoring System}

Three-dimensional DC method using electrical resistivity tomography technology for data processing and mapping. Which usually use the existing DC equipment and detection methods by optimizing and improving the construction method, and encrypting the sampled data to obtain the $3 \mathrm{D}$ data. The method collects more data, the information is large, the accuracy is high and the measured 3D data can reflect the resistivity distribution of underground 3D space[6-7].Limited to the roadway space, A three dimensional DC resistivity observation system with fixed source is proposed, as shown in Figure 1. Electrode A and B are arranged in the two tunnels, about 30 50 meters from the coal mining machinery, which connect the host through cable. The position of the electrode A and $\mathrm{B}$ move backward along with coal mining, and measuring electrodes which designed according to the requirements are layouted in the new mining area, so that the observed real-time potential is transmitted to the computer through wireless transmission.

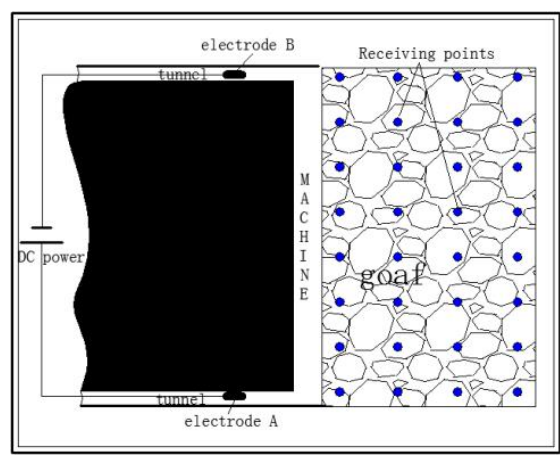

Fig. 1. System of 3D DC resistivity method for monitoring 


\section{B. Forward Theory}

In the three-dimensional cartesian coordinates, the boundary value problem of partial differential equation which can be satisfied by the basic theory of stable current field can be get if power point is located at $A\left(x_{A}, y_{A}, Z_{A}\right)$, and its current is $\mathrm{I}[8-10]$.

$$
\begin{aligned}
& \left\{\begin{array}{l}
L u \equiv \frac{\partial}{\partial x}\left(\sigma \frac{\partial u}{\partial x}\right)+\frac{\partial}{\partial y}\left(\sigma \frac{\partial u}{\partial y}\right)+\frac{\partial}{\partial z}\left(\sigma \frac{\partial u}{\partial z}\right)=f \\
\left.\frac{\partial u}{\partial n}\right|_{\Gamma_{D}}=0 \\
\left.\left(\frac{\partial u}{\partial n}+\gamma u\right)\right|_{\Gamma_{T}}=0
\end{array}\right. \\
& f=-I \cdot \delta\left(x-x_{A}\right) \cdot \delta\left(y-y_{A}\right) \cdot \delta\left(z-z_{A}\right)
\end{aligned}
$$

$\Gamma_{n}$ : Roadway border, $\Gamma_{T}$ : Underground infinite boundary,

$\gamma=\cos \theta / r, \theta$ : radial vector from point source to the boundary, it is the angle between $\vec{r}$ and $\vec{n}$ (normal vector).

\section{FORWARD MODELS}

Set up the numerical simulation model parameters as follows: Workface width: 200 meters, Top, bottom thickness: 100 meters, Seam thickness: 10 meters, Top, bottom resistivity: $100 \Omega$, goaf: $80 \Omega$, coal: $1000 \Omega$, abnormal: $0.01 \Omega$. Abnormal body length and width: $20 \mathrm{~m}$. The parameters of different positions of abnormal body are shown in Table 1.The distence between the top surface of anomalous body and floor is 20 meters. On the horizontal, the center of the abnormal body are located on the vertical line of the power. 4 models (A1 A4) are calculated as the distence between the abnormal body to the left boundary of the calculation area is variable $S_{1}$. On the vertical, 4 models (A5 A8) are calculated as the distence between the abnormal body to the lower boundary of the calculation area is variable $S_{2}$. Its schematic as shown in Figure 2. Anomalies depth position parameters are shown in Table 2. When the anomaly center is located on the vertical line of the power, 4 models (B1 B4) are calculated as the distence between the top surface of abnormal to the floor is variable $\mathrm{H}$. When the abnormal center is close to the negative side of the power, 4 models (B5 B8) are calculated as the distence between the top surface of abnormal to the floor is variable H.Its schematic as shown in Figure 3.

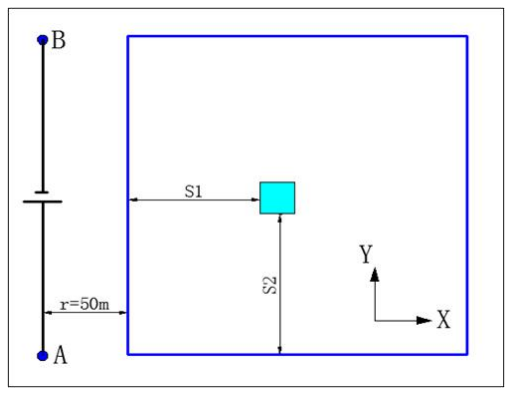

Fig. 2. Anomalies of different positions in the same plane

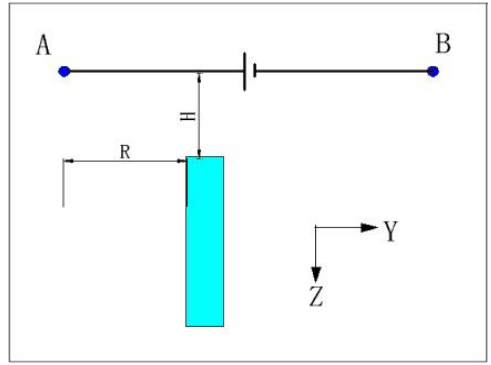

Fig. 3. Anomalies of different depths

TABLE I. THE POSITION PARAMETERS OF ANOMALIES

\begin{tabular}{ccccc}
\hline $\begin{array}{c}\text { models } \\
\text { distance }\end{array}$ & A1 & A2 & A3 & A4 \\
\hline $\mathrm{S}_{1} / \mathrm{m}$ & 10 & 50 & 90 & 130 \\
\hline $\mathrm{S}_{2} / \mathrm{m}$ & 90 & 90 & 90 & 90 \\
\hline $\begin{array}{c}\text { models } \\
\text { distance }\end{array}$ & A5 & A6 & A7 & A8 \\
\hline $\mathrm{S}_{1} / \mathrm{m}$ & 90 & 90 & 90 & 90 \\
\hline $\mathrm{S}_{2} / \mathrm{m}$ & 30 & 60 & 120 & 150 \\
\hline TABLE II. & THE DEPTH PARAMETERS OF ANOMALIES \\
\hline $\begin{array}{c}\text { models } \\
\text { distance }\end{array}$ & B1 & B2 & B3 & B4 \\
\hline $\mathrm{H} / \mathrm{m}$ & 40 & 30 & 20 & 10 \\
\hline $\mathrm{R} / \mathrm{m}$ & 90 & 90 & 90 & 90 \\
\hline $\begin{array}{c}\text { models } \\
\text { distance }\end{array}$ & B5 & B6 & B7 & B8 \\
\hline $\mathrm{H} / \mathrm{m}$ & 40 & 30 & 20 & 10 \\
\hline $\mathrm{R} / \mathrm{m}$ & 140 & 140 & 140 & 140 \\
\hline
\end{tabular}

The potential distribution of abnormal body in different position is shown in Figure 4. On the horizontal, As the center of the abnormal body located on the vertical line of the power, there are both the maximum and minimum points on the contour map. The position of the extreme point is symmetrical distribution on the vertical distribution of the power, and also moves along with the lateral movement of the abnormal body, the distance from the extreme point to the left boundary of the calculation area: $S_{1} \approx S_{1}$. Due to the impact of abnormal depth and volume effect, the distence of the two poles is different from the abnormal body length. The absolute value of the extreme value decreases with the distance to the source. On the vertical, when the anomalous body is close to the positive electrode of source, only the minimum point appears on the contour map. And the distance from this point to the lower boundary of the calculation area: $S_{2} \approx S_{2}$ when the anomalous body is close to the negtive electrode of source, only the maximum point appears on the contour map. And the distance from this point to the lower boundary of the calculation area: $S_{2} \approx S_{2}+L$ (L: abnormal body length, 20 meters). The center position of the extreme point is determined by abnormal body edge which located near the source pole. The effect of abnormal potential is gradually dominant, and the absolute value of extreme value is increased with the abnormal position move closer to one pole of the source. 


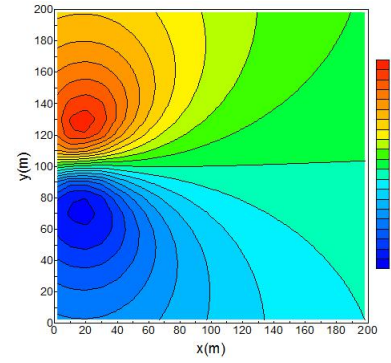

A1

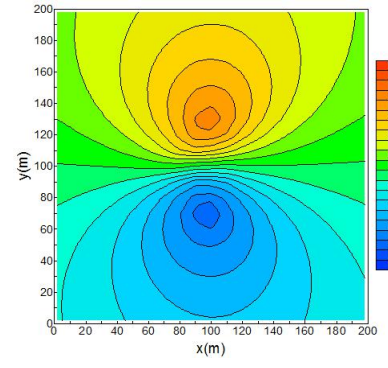

A3

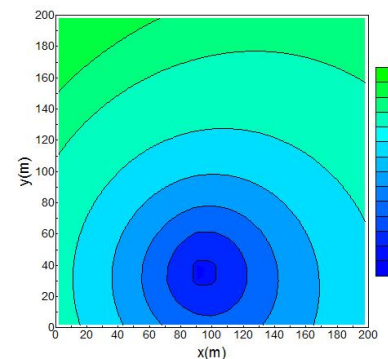

A5

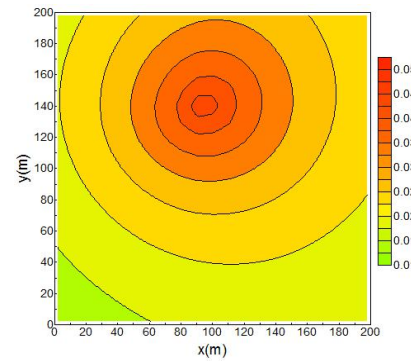

A7

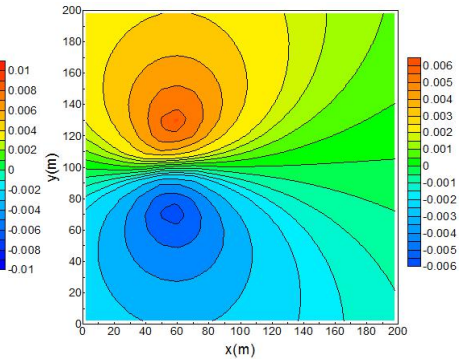

A2

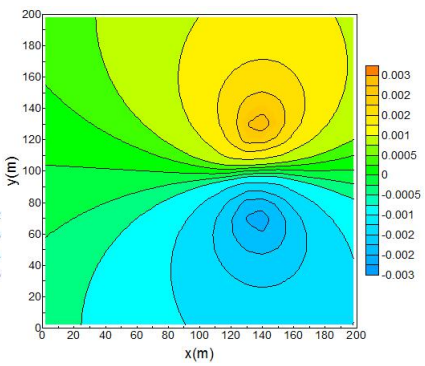

A4

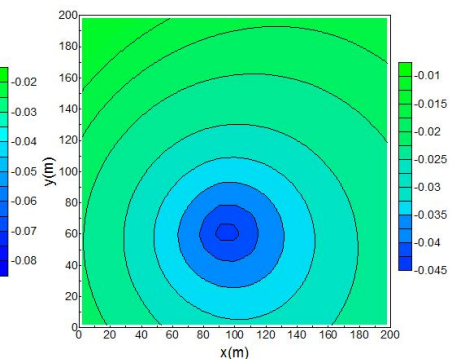

A6

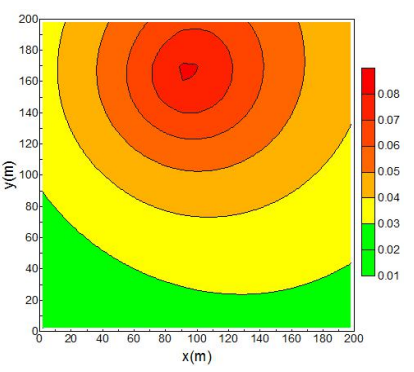

A8

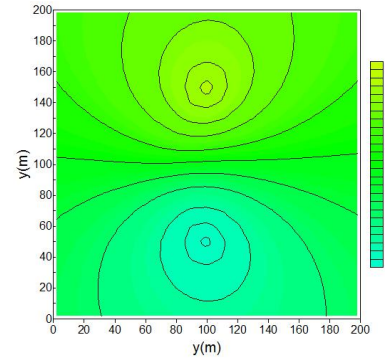

$\mathrm{B} 1$

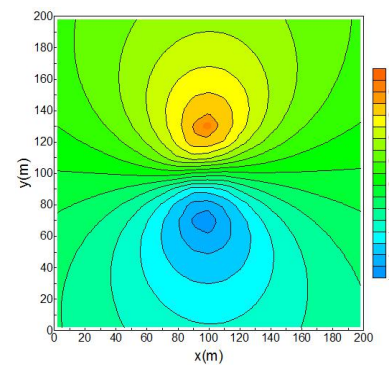

B3

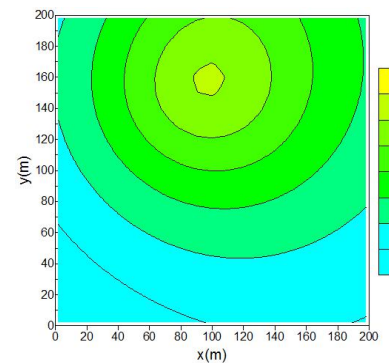

B5

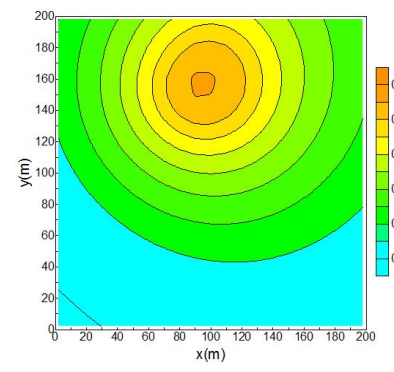

B7

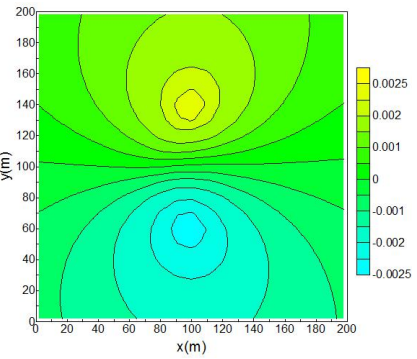

B2

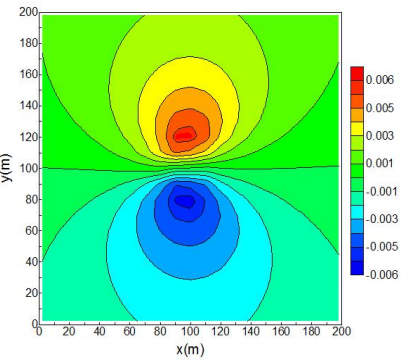

B4

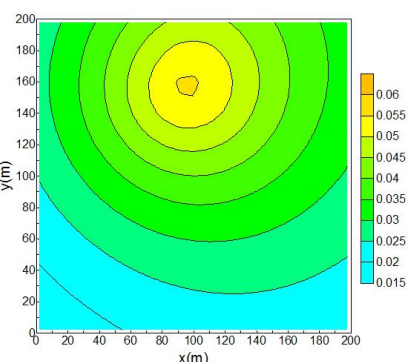

B6

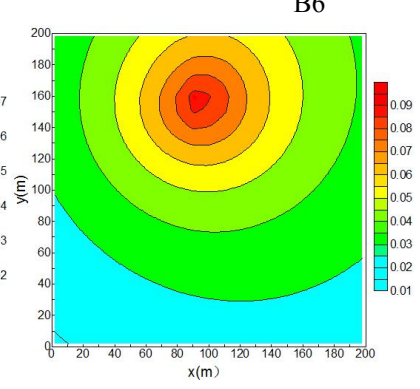

B8
Fig. 4. Potential contour of Anomalies in different position of bottom plate

The potential distribution of abnormal body in different depths is shown in Figure 5. When the center of the abnormal body located on the vertical line of the power $(R=90 \mathrm{~m})$, there are both the maximum and minimum points on the contour map. The position of the extreme point is symmetrical distribution on the vertical distribution of the power, the distence of the two poles has the consistent change trend with $\mathrm{H}$. The absolute value of the extreme value increases with the abnormal depth becomes shallow. When the abnormal center is biased towards the negative side of the power $(\mathrm{R}=140 \mathrm{~m})$, there is only one maximum point on the contour map.Its plane position is in agreement with the plane coordinate of the anomaly, which does not change with the change of the depth of the anomaly. Also the absolute value of the extreme value increases with the abnormal depth becomes shallow.

\section{CONCLUSIONS}

It can be concluded that the anomalous bodies in different locations and different depths can reflect their positions on the contour maps of the abnormal potential easily,through forward modeling of multiple models in the three-dimensional DC resistivity observation system. Thus, this characteristic can be used for real-time monitoring the lagging water inrush from floor. This provides a theoretical reference for the prevention of mine water.

\section{REFERENCES}

[1] Liu S C. Mechanism of water inrush from coal seam floor and continuous survey of fractured zones in coal seam floor. [Doctor's thesis]. 2008, Jiangsu: China University of Mining and Technology. 
[2] Shi L Q, Han J. Mechanism and prediction of water inrush from floor. 2004, Xuzhou: China University of Mining and Technology Press.

[3] Ren Z Y, Tang J T. Finite element modeling of 3-D DC resistivity using locally refined unstructured meshes. Chinese Journal of Geophysics (in Chinese), 2009, 52(10):2627-2634.

[4] Qiang J K, Luo Y Z. The resistivity FEM numerical modeling on 3-D undulating topography. Chinese Journal of Geophysics (in Chinese), 2007, 50(5):1606-1613.

[5] Yue J H, Li Z D. Mine DC method and its application in the detection of water inrush from coal seam floor. Journal of China University of Mining \& Technology (in Chinese), 1997, (01):94-98.

[6] Panissod C, Hesse A, Jolivet A, et al. Recent developments in shallowdepth electrical and electrostatic prospecting using mobile arrays[J]. Geophysics , 2012, 63(5):1542-1550.
[7] Zhang Y W, Yan J Y, Zhang K, et al. Review of distributed 3D DC/IP method. Progress in Geophysics (in Chinese), 2015, (04).

[8] Xu S Z, Liu B, Ruan B Y. The finite element method for solving anomalous potential for resistivity surveys. Chinese Journal of Geophysics (in Chinese), 1994, (S2):511-515.

[9] Mallick K, Jain S C. Resistivity sounding on a layered transitional earth. Geophysical Prospecting, 1979, 27(4):869-875.

[10] Liu Z X. Research on 3D Finite Element Simulation Method for Mine DC Electrical Method [Master's thesis]. Jiangsu: China University of Mining and Technology,2002. 